\title{
Ambulance Calling System using Heart Rate Sensor in Smart Band
}

\author{
V. Sai Harsha, U. Kumaran
}

\begin{abstract}
Crisis medicinal reaction in India is lingering behind other nations. This is incompletely a direct result of absence of innovation usage at ground zero. To address the issue, we are presenting keen emergency vehicle framework. It would take India to focused position in crisis benefits far and wide. In the course of the most recent couple of years there is a progressive improvement in the field of Internet of Things (IoT). It very well may be utilized consistently and broadly in vast number of end framework where subset of a lot of information can be gotten to and prepared effectively and capably. IoT and cell phone advancements helps in structure a stage which serves each cell phone client. It very well may be utilized consistently to fortify the crisis medicinal reaction by means of smart band rescue framework, as IoT can likewise be utilized broadly in vast number of end framework where a lot of information can be gotten to and handled effectively and intensely. IoT and brilliant gadgets helps in structure a stage which serves each smart gadget client where a brilliant band will constantly center around observing heart thumps of a individual wearing this band. This information will be gathered through an application and send to a brought together database, where it will get separated for any anomalies, and whenever found any then that individual will be educated and if necessary an emergency vehicle will be dispatched to their whereabouts. If there should be an occurrence of any crisis Mobile GPS is consequently activated and the message is sent to the Server by means of portable GSM. The Server will ascertain the closest way to achieve the Ambulance and furthermore sends a ready SMS to the relatives.
\end{abstract}

Key Words: GPS, Pulse Rate, identification, Emergency, Public Safety.

\section{INTRODUCTION}

Getting ambulance inside least measure of time, giving appropriate treatment to the patient so that the possibility of enduring increments in basic condition. We can beat these impediments by utilizing forthcoming innovation like IoT i.e., Internet of Things. Different equipment gadgets can be associated with one another by means of wired and remote systems administration instruments and programming executions. Utilization of different APIs can impart between the server what's more, customer end which is actualized in this task. APIs are structured so that time multifaceted nature will be limited broadly.

Revised Manuscript Received on July 13, 2019

V.Sai Harsha, UG Scholar, Saveetha School of Engineering, Saveetha Institute of Medical and Technical Sciences, Thandalam, Chennai, Tamilnadu, India- 602105

U. Kumaran, Associate Professor, Faculty of Engineering, Saveetha School of Engineering, Saveetha Institute of Medical and Technical Sciences, Thandalam, Chennai, Tamilnadu, India-602105

Expectedly amid a crisis, an injured individual or the victim's guardian calls rescue vehicle dispatch administration by dialling the helpline number. The emergency vehicle dispatcher requests the definite location and calls the accessible rescue vehicle to go to that specific area. This process devours time. An individual need to first make a call and hold up until the call interfaces and after that direct the rescue vehicle dispatcher about the area of mishap or crisis. The dispatcher then cautions the rescue vehicle close to the area of the person in question and aides the emergency vehicle to the person in question. If there should be an occurrence of crisis, consistently is essential for sparing an actual existence and one can't afford to hold up longer. The need of a framework that can lessen this time where the data of the injured individual flows from the unfortunate casualty to dispatcher and dispatcher to rescue vehicle. This explore work proposes a cell phone application that can sidestep the need of manual dispatcher and empower the direct communication between closest emergency vehicle and the injured individual along these lines, lessening the postponement in correspondence and at last the landing of the emergency vehicle to safeguard the sufferer. By utilizing a straightforward cell phone application, the sufferer can legitimately interact with adjacent emergency vehicle. This time slack between the event of accident and hint to the adjacent clinic must be decreased to spare all the more valuable life of unfortunate casualties. Different existing ideas and some blasting advances are mixed in this exploration work, to outline the sheltered and secure framework for patients emergency vehicle administration. Worldwide Positioning System (GPS) utilizes satellite for following the versatile empowered with GPS module. Circling satellites are utilized to sends the scope and height of the following focused on GPS empowered gadget utilizing the procedure of Trilateration. This epic research paper features the required headway and arrangement, to spare patients life by adding insight to the current emergency vehicle framework for example event of mishap is followed utilizing GPS and it is hinted to the close-by rescue vehicle administration to the medical clinic. There are essentially two modules in this Smart Ambulance System, for example Vehicle module which is put in the patient's vehicle with GSM and GPS empowered gadgets and recipient module which is a cell phone to get Short Message Service of the mishap area to the adjacent medical clinic for rescue vehicle administration. 
The required modules are, Global Positioning SystemGPS for accident area data, GSM Transmitter and Receiver, Vibration Detecting for panic.

\section{LITERATURE SURVEY}

In this paper, the structure and improvement of wearable biosensor frameworks for wellbeing observing has earned loads of consideration in mainstream researchers and the industry amid the most recent years. Chiefly roused by expanding medicinal services costs and impelled by later mechanical advances in smaller than normal bio detecting gadgets, shrewd materials, microelectronics, and remote correspondences, the constant development of wearable sensor based frameworks will possibly change the eventual fate of human services by empowering proactive individual wellbeing the executives furthermore, omnipresent observing of a patient's wellbeing condition. [1]

Web of Things (IoT) is a system of sensors, actuators, portable and wearable gadgets, just things that have handling and correspondence modules and can interface with the Internet. In a couple of years time, billions of such things will begin serving in numerous fields inside the idea of IoT. Self setup, self-governing gadget expansion, Internet association and asset confinement highlights of IoT makes it be profoundly immediately the assaults. Refusal of Service (DoS) assaults which have been focusing on the correspondence systems for a long time, will be the most hazardous dangers to IoT systems. [2]

A coordinated GPS-GSM framework is proposed to follow vehicles utilizing Google Earth application. The remote module has a GPS mounted on the moving vehicle to recognize its current position, and to be exchanged by GSM with other parameters procured by the vehicle's information port as a SMS to a beneficiary station. [3]

Wellbeing checking frameworks have quickly developed as of late, and smart frameworks have been proposed to screen quiet present wellbeing conditions, in our proposed and executed framework, we center around checking the patient's circulatory strain, and his body temperature. In light of a decade ago measurements of medicinal records, demise rates due to hypertensive coronary illness, demonstrates that the circulatory strain is a urgent hazard factor for atherosclerosis and ischemic heart maladies; hence, preventive measures ought to be taken against hypertension which give the capacity to follow, follow what's more, spare patient's life at suitable time is a fundamental need for humanity. The goal of this work is giving an compelling application for Real Time Health Monitoring and Following. The framework will follow, follow, screen patients and encourage dealing with their wellbeing; so effective therapeutic administrations could be given at fitting time [4]

The Internet of Things (IoT) frameworks empower the correspondence of unfriendly suite of gadgets and items, anyway it is realized that security is one of the major issues in these frameworks. This is basically because of the way that IOT gadgets work with very constrained computational power and vitality spending plan and traditional cryptographic strategies will be excessively costly. To this end, we propose a novel crossover security convention and exhibit its reasonableness through a genuine time emergency vehicle tracking application. [5]

Web of Things (IoT) and distributed computing plays a essential job in the present Tele-observing wellbeing framework. This framework monitors patient's physiological parameters through accumulation of body sensors' information utilizing Raspberry Pi board. The patient's wellbeing card are created by the specialists and showed on a site page where specialists and patients can get to and impart each other without physical nearness [6].

Utilizing distributed computing, the information can be put away, refreshed also, got to from anyplace on the planet. It is very appropriate for rustic regions where therapeutic offices are most certainly not accessible. In Remote wellbeing checking framework utilizing IoT, Body remote sensor Network (BWSN) is utilized to transmit the patients' wellbeing parameters [7].

The particular medicinal services checking framework for old individuals is a developing need in the maturing populace world. This framework performs fundamental wellbeing checkups by estimating the body parameters normally furthermore, report the information to the specialists. The outcome information are at that point showed as articulations in a web application where specialists and patients can communicate with one another [8].

Framework gathers information of different body parameters through Biosensors, wearable gadgets and shrewd materials and it transmits the information to focal hub server safely through Cipher content Policy Attribute Based Encryption (CP-ABE) strategy. Thus, the server shares the gathered information to the medical clinics for further treatment [9].

IoT based Smart social insurance with the assistance of smart gadgets and items improves the social insurance checking framework adequately, hence by lessening the wasteful aspects of existing human services framework. Keen gadgets with new also, updated advancements improves the information exactness to be gathered, ongoing availability of patient's condition, savvy joining of information gathered, keeping up the incorporated information adroitly through cloud administration[10]

\section{PROPOSED SYSTEM}

The framework will contain three principle units. The wearable gadget will worn by the client. This gadget will screen the client heart beat and will track the client. The second unit will be an android portable, this unit will be associated with the wearable gadget by Bluetooth. The third unit is about broadcasting the alarm message by SMS from android portable.

To take care for the older individual a smart band will be presented which will screen all the wellbeing issues, for example, beat, circulatory strain and essentially it will screen the heart pulsates of the individual. This smart band can be effectively wearable on the wrist of our hand and it will be associated with cell phones all the time. The information gathered from this 
band will be effectively exchanged with the assistance of gave application with the assistance of cell phones. Smart band will screen the individual $24 X 7$ and will keep on sending the information. This substantial measure of information will be send to a brought together database, where vast sum of information will get gathered in an orderly manner. All the procedure will happen naturally with the assistance of IoT i.e., Internet of Things.

The dispatched emergency vehicle will know about the area of the individual with the assistance of brilliant band or the cell phone application. The client or the guardian of that individual will almost certainly track the area of the dispatched rescue vehicle on their cell phones with the assistance of Global Positioning Framework (GPS) and Google maps APIs on this application.

\section{Architecture}

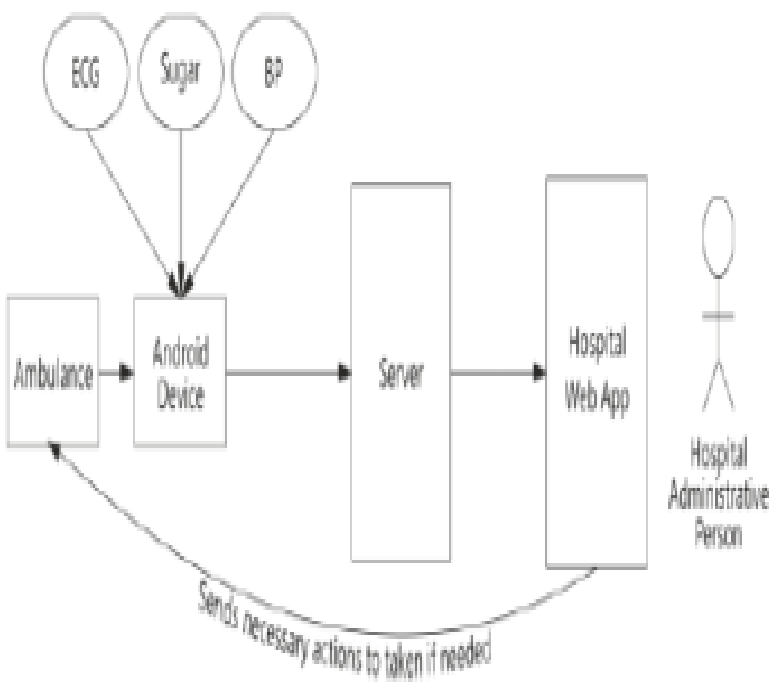

Fig. 1 System Architecture

The above architecture helps us to understand more clearly how the information will flow step by step and the pictorial view of the idea represented in this paper. Here in the above architecture diagram the work flow between ambulance calling and patient is shown.

\section{Results}

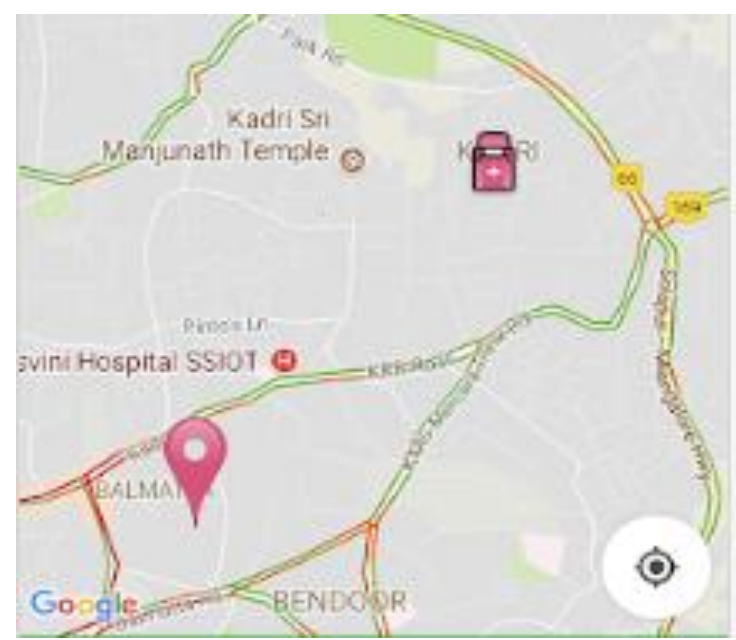

Fig. 2 Location of ambulance and the patient
The above image displays the real time location of the ambulance using GPS.

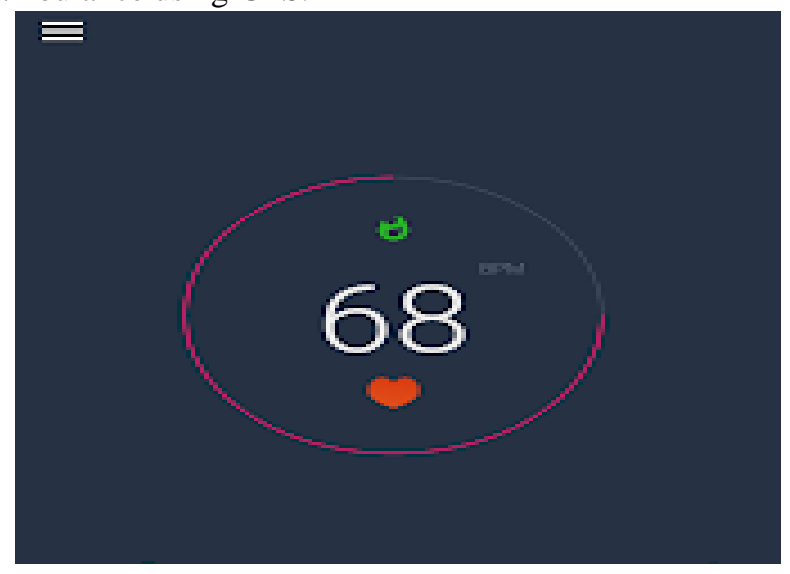

Fig. 3 Heart beat of the patient

The above image shows the heartbeat of the patient using the smart band.
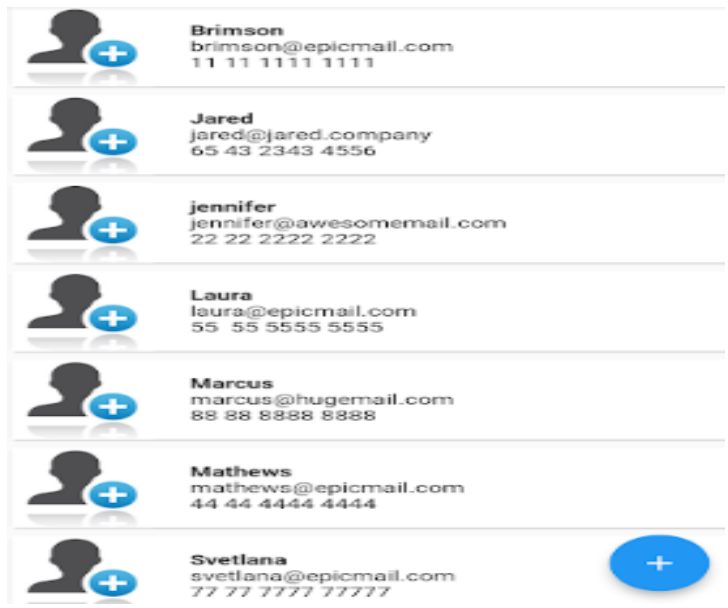

Fig. 4 List of contacts

The above image shows the list of contacts that gets the panic alert.

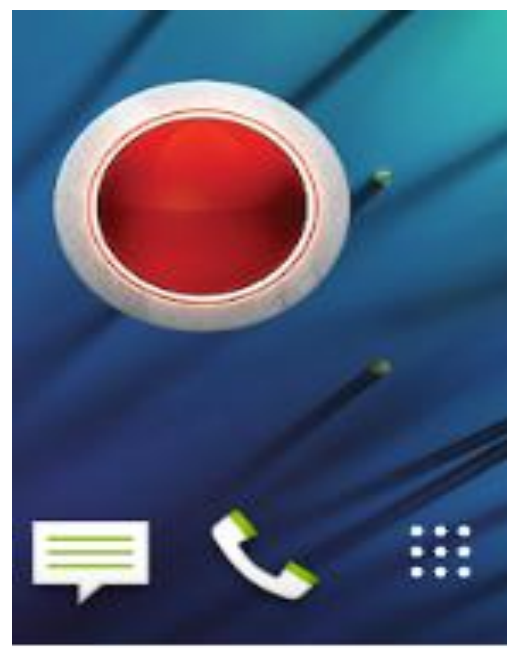

Fig. 5 Panic button on home screen

The above image shows the panic button on home screen which triggers the functionality of ambulance calling.

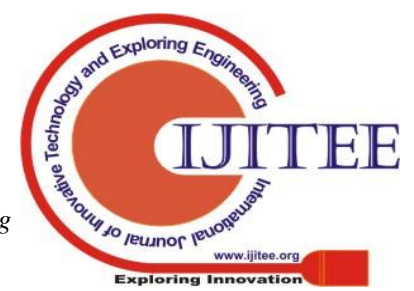




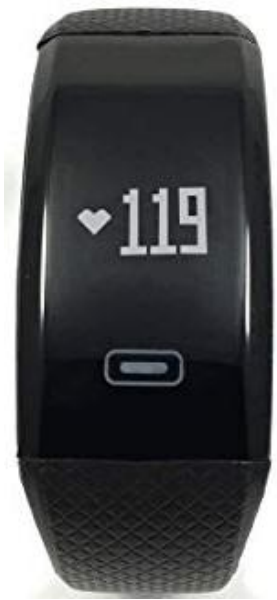

Fig. 6 Smart band heart rate

\section{CONCLUSION}

In this paper, a thought is proposed for sparing a patient's life in a quicker manner conceivable. It is valuable for clients if there should arise an occurrence of crises as it spares time. With this Application, the emergency vehicle can come to the patients as area is given through the application and can give essential hardware required to the patient's wellbeing. Data about the medical clinics gave helps in getting the proper emergency clinic which is reasonable for the patient's treatment. The live feed information sent through the emergency vehicle to the clinic helps in monitoring patient's wellbeing subtleties and reaches the clinic with no time slack. Sending patient's wellbeing data to the clinics helps the medical clinic staff to get the important pre-necessities with respect to the patient's treatment. Subsequently it lessens the time multifaceted nature and serves to give quicker medicinal administrations. The other main feature of this application is to send the panic alert to the contacts along with the GPS location. And a panic button on the home screen helps the user to trigger the ambulance calling system on one click. This helps the user to use the service when the wearable device is not with the user.

\section{REFERENCES}

1. Ateeth Kumar Thirukkovulur1, Harikrishna Nandagopal, Vigneesh Parivallal," Intelligent Vehicle Control Based On Identification Of Road And Traffic Signal Operated RFID Transponders, International Conference On Advances In Electrical and Electronics Engineering (ICAEE'2012) Penang, Malaysia.

2. Rajat \&Nirbhay Kumar (2007) "RFID Resolution: Your cars will be tagged", The Economics Times, 25 September.

3. Allwinkle, S. \& Cruickshank, P. (2011) Creating smart-er cities: An overview, Journal of Urban Technology, 18(2), 1-16.

4. Internet of things for Smart Cities. Andrea Zanella, Senior Member, IEEE, Nicola Bui, Angelo Castellani, Lorenzo Vangelista, Senior Member, IEEE, and Michele Zorzi, Fellow, IEEE. IEEE INTERNET OF THINGS JOURNAL, VOL. 1, NO. 1, FEBRUARY 2014

5. An IoT-Aware Architecture for Smart Healthcare Systems. Luca Catarinucci, Danilo de Donno, Luca Mainetti, Luca Palano, Luigi Patrono, Maria Laura Stefanizzi, and Luciano Tarricone, IEEE INTERNET OF THINGS JOURNAL, VOL. 2, NO. 6, DECEMBER 2015.

6. Outrage at how the 911 emergency service 'failed New York' during Hurricane Sandy. Available from: http://www.dailymail.co.uk/news/article-2235113/Hurricane-Sandy-
Outrage-911-emergency-service-failed-NewYork html\#ixzz3tFDoNQhRc1

7. Provincial Disaster Management Authority. Available from: http://www.pdma.gov.pk/

8. Traffic Accidents. Available from: http://www.pbs.gov.pk/content/tra $\square$ c-accidents-annual

9. Mundasad S. Patients 'face long queues in ambulances outside A\&E'. Available from:http://www.bbc.com/news/health-28706796

10. S. Jagadeeshwaran, N. Dinesh, Chembur, Mumbai, INDIA Computer Technology Department, VeermataJijabai Technological Institute, Matunga, Mumbai, INDIA "Automatic Ambulance Rescue System", April 2012.

11. Siva Kumar Avula, Cheng Siong Lim, Shubhangi C Deshmukh, IEEE "Impact of Ambulance Dispatch Policies on Performance of Emergency Medical Services", December 2011.

12. Ch. Sandeep Kumar Subudhi, S.Sivanandam International Journal of Interdisciplinary and Multidisciplinary Studies Intelligent Wireless Patient Monitoring and Tracking System (Using Sensor Network and Wireless Communication",Page No.-97-104, Volume 1 , Issue 2014.

13. Y. Sudhindra.F, Annarao.S.J, Vani.R.M, P.V. Hunagund International Journal of Research in Engineering and Technology A GSM Enabled Real Time Simulated Heart Rate Monitoring Control System", Page No. 6-10 Volume: 03, May 2014

14. Cheng Siong Lim, RosbiMamat and Thomas Brunl Senior Member IEEE Impact of Ambulance Dispatch Policies on Performance of Emergency Medical Services”, 2011.

15. Ricquebourg Vincent, David Menga, David Durand, Bruno Marhic, Laurent Delahoche, Christophe Loge, "The smart home concept: our immediate future", E-Learning in Industrial Electronics 2006 1ST IEEE International Conference on, pp. 23-28, December 2006.

16. Malhi Karandeep, Subhas Chandra Mukhopadhyay, Julia Schnepper, Mathias Haefke, Hartmut Ewal, "A Zigbeebased wearable physiological parameters monitoring system", Sensors Journal IEEE, vol. 12, no. 3, pp. 423-430, March 2012. 